\title{
Association Between Visceral Hypersensitivity in Irritable Bowel Syndrome, Intestinal Microbiota, and Mast Cells: How to Detect Mast Cells Using Confocal Microscopy
}

TO THE EDITOR: Although the pathophysiology of irritable bowel syndrome (IBS) is multifactorial, visceral hypersensitivity $(\mathrm{VH})$ is of the most importance. The possible underlying mechanism of $\mathrm{VH}$ was suggested by a recently published study in the Journal of Neurogastroenterology and Motility entitled "The role of intestinal microbiota and mast cell in a rat model of visceral hypersensitivity" by $\mathrm{Li}$ et al. ${ }^{1}$ The authors showed the association between mast cells and intestinal microbiota in an animal model of VH. In the VH rat model induced by acetic acid enema with partial limb restraint method, mast cell numbers and their degranulation significantly increased compared with the controls. Those increases were reduced by administration of ketotifen or probiotic VSL\#3, which restored Clostridium sensu stricto 1 abundance. The study suggests that IBS could be detected and managed by observing or manipulating mast cells and intestinal microbiota. Therefore, we thought to image mast cells with no need of using conventional methods including immunohistochemistry for either tryptase or ckit or electron microscopy. We would like to introduce cases of intestinal mast cell images using confocal microscopy without special staining.

This study was approved by the Hanyang University institutional review board (2020-01-033-003) and written informed consents were obtained from the study patients before participation. We obtained intestinal tissues (rectal mucosa) during colonoscopy from patients with diarrhea-predominant IBS who were diagnosed by symptom-based criteria. The rectal mucosa samples were sectioned at $2 \mu \mathrm{m}$ in thickness, and prepared on 3 slides. The first and the third layers are stained with toluidine blue and with the rabit polyclonal anti-CD117 (A4502; Dako Cytomation, Carpinteria, CA, USA), respectively. And the second layer (sandwiched by 2 stained layers) is applied for the confocal imaging without any staining. The unstained slides were observed by scattering-type confocal microscopy (SCM) after locating where to be able to find mast cells (the same areas with many crypts in each slide). The SCM is a specialized microscopy, where the source of the input light was a cw-mode $532 \mathrm{~nm}$ laser, and the output signal from the mast cells was detected using a photomultiplier tube. The images from the output signal were acquired at a frame rate of up to $2 \mathrm{~Hz}$ and a resolution of $1024 \times 1024$ pixels. We observed unstained slides and 2 adjacent slides immunohistochemically stained with either toluidine blue or c-kit (CD 117). We could determine by SCM whether candidate cells have the unique features of the average size and distributional shape of internal granules and nucleus in mast cells. Then, when positively stained by both c-kit and toluidine blue, they were considered as a mast cell; or as a non-mast cell when stained by neither c-kit nor toluidine blue (Figure). The confocal microscopy without fluorescent dyes differentiated 43 mast cells from 52 candidate cells and 37 non-mast cells from 39 candidate cells. Thus, the correct detection rate was estimated to be $88 \%$ (80/91). Limitations are that it is not yet possible to accurately differentiate degranulated mast cells from non-mast cells and to be directly applied to clinical practice because of problems in device equipment and potential intra- and inter-observer differences.

The role of mast cells in the pathophysiology of IBS has been 

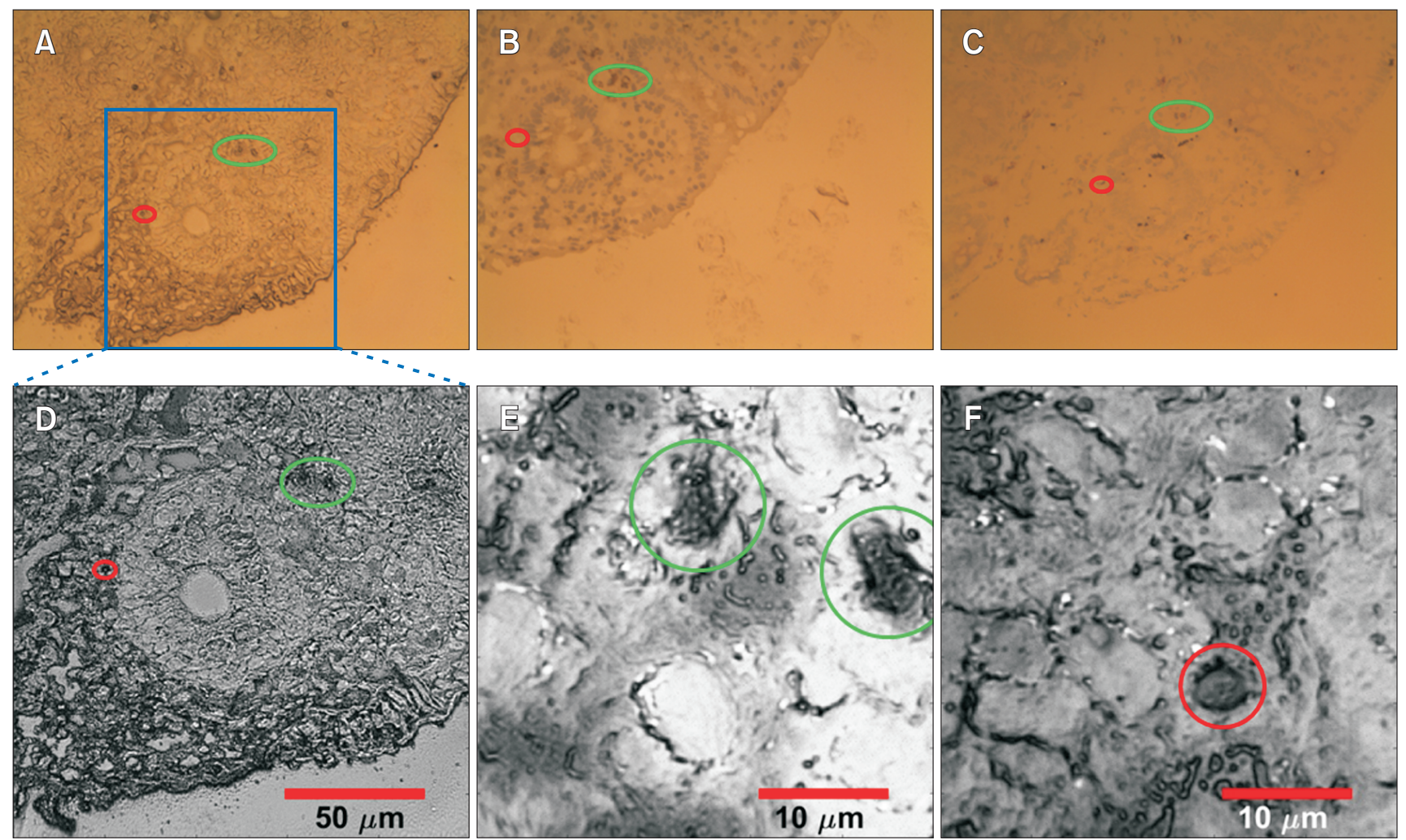

Figure. Representative photomicrographs showing intestinal mast cells in (A) unstained, (B) c-kit-stained, and (C) toluidine blue-stained slides. Cells within green and red circles are mast cell and a non-mast cell, respectively. Scattering confocal microscopy image of the selected area (blue square in [A]) obtained with a magnification of $\times 20$ (D), and the cases of mast cell (E) and the case of a non-mast cell (F) with a magnification of $\times 100$.

argued. $^{2}$ However, studies have demonstrated that mast cells can modulate sensory and motor nerves, ${ }^{3-6}$ increase epithelial secretion and intestinal permeability, ${ }^{7,8}$ and affect gut immunity and the braingut axis. ${ }^{9}$ These suggest that intestinal mast cells may play a pivotal role in the pathophysiology of IBS and thus, imaging mast cells might be used in diagnosing IBS. ${ }^{10}$ Although we could detect mast cells in patients with IBS using SCM, further research is needed to combine with confocal endomicroscopy for in vivo detecting mast cells and their changes after manipulating intestinal microbiota in IBS patients with $\mathrm{VH}$.

Kang Nyeong Lee, ${ }^{1}$ Jiwon Lee, ${ }^{2}$ Juil Hwang, ${ }^{2}$ Chungha Lee, ${ }^{2}$ Ji Sook Kim, ${ }^{3}$ Seok Ho Song, ${ }^{2}$ Oh Young Lee, ${ }^{1 *}$ and Kwang-Geol Lee ${ }^{2 *}$ ${ }^{\prime}$ Department of Internal Medicine, Hanyang University Hospital, Seoul, Korea; 'Department of Physics, Hanyang University, Seoul, Korea; and ${ }^{3}$ Department of Pathology, Hanyang University Hospital, Seoul, Korea

1. Li YJ, Li J, Dai C. The role of intestinal microbiota and mast cell in a rat model of visceral hypersensitivity. J Neurogastroenterol Motil 2020;26:529-538.
2. Zhang L, Song J, Hou X. Mast cells and irritable bowel syndrome: from the bench to the bedside. J Neurogastroenterol Motil 2016;22:181-192.

3. Buhner S, Braak B, Li Q, et al. Neuronal activation by mucosal biopsy supernatants from irritable bowel syndrome patients is linked to visceral sensitivity. Exp Physiol 2014;99:1299-1311.

4. Xu XJ, Zhang YL, Liu L, Pan L, Yao SK. Increased expression of nerve growth factor correlates with visceral hypersensitivity and impaired gut barrier function in diarrhoea-predominant irritable bowel syndrome: a preliminary explorative study. Aliment Pharmacol Ther 2017;45:100114.

5. Traver E, Torres R, de Mora F, Vergara P. Mucosal mast cells mediate motor response induced by chronic oral exposure to ovalbumin in the rat gastrointestinal tract. Neurogastroenterol Motil 2010;22:e34-e43.

6. Balestra B, Vicini R, Cremon C, et al. Colonic mucosal mediators from patients with irritable bowel syndrome excite enteric cholinergic motor neurons. Neurogastroenterol Motil 2012;24:1118-e1570.

7. Lee H, Park JH, Park DI, et al. Mucosal mast cell count is associated with intestinal permeability in patients with diarrhea predominant irritable bowel syndrome. J Neurogastroenterol Motil 2013;19:244-250.

8. Wilcz-Villega EM, McClean S, O'Sullivan MA. Mast cell tryptase reduces junctional adhesion molecule-A (JAM-A) expression in intestinal epithelial cells: implications for the mechanisms of barrier dysfunction in 
irritable bowel syndrome. Am J Gastroenterol 2013;108:1140-1151.

9. Lee KN, Lee OY. The role of mast cells in irritable bowel syndrome. Gastroenterol Res Pract 2016;2016:2031480.

10. Boyer J, Saint-Paul MC, Dadone B, et al. Inflammatory cell distribution in colon mucosa as a new tool for diagnosis of irritable bowel syndrome: a promising pilot study. Neurogastroenterol Motil 2018;30.
Financial support: This work was supported by the Basic Science Research Program through the National Research Foundation (NRF) of Korea and funded by the Ministry of Science and ICT (Grant No. 2020R1A2C1010014).

\section{Conflicts of interest: None.}

Author contributions: Concept: Oh Young Lee and Kwang-Geol Lee; supervision: Oh Young Lee, Kwang-Geol Lee, and Seok Ho Song; resources: Seok Ho Song and Kwang-Geol Lee; materials: Ji Sook Kim and Kwang-Geol Lee; data collection and/or processing: Jiwon Lee, Ji Sook Kim, Juil Hwang, and Chungha Lee; analysis and/or interpretation: Jiwon Lee, Kang Nyeong Lee, and Ji Sook Kim; literature search: Jiwon Lee, Kang Nyeong Lee, Juil Hwang, and Chungha Lee; writing manuscript: Jiwon Lee and Kang Nyeong Lee; and critical review: Oh Young Lee and Seok Ho Song. Kang Nyeong Lee and Jiwon Lee contributed equally to this work, and *Oh Young Lee (leeoy@hanyang.ac.kr) and Kwang-Geol Lee (kglee@hanyang.ac.kr) are equally responsible for this work. 